\title{
Synergistic inhibitory effect of gemcitabine and angiotensin type-1 receptor blocker, losartan, on murine pancreatic tumor growth via anti-angiogenic activities
}

\author{
RYUICHI NOGUCHI, HITOSHI YOSHIJI, YASUHIDE IKENAKA, TADASHI NAMISAKI, \\ MITSUTERU KITADE, KOSUKE KAJI, JUNICHI YOSHII, KOJI YANASE, MASAHARU YAMAZAKI, \\ TATSUHIRO TSUJIMOTO, HIDETO KAWARATANI and HIROSHI FUKUI
}

Third Department of Internal Medicine, Nara Medical University, Nara 634-8522, Japan

Received March 11, 2009; Accepted May 8, 2009

DOI: $10.3892 /$ or_00000445

\begin{abstract}
Pancreatic cancer is one of the leading causes of cancer death, and represents a challenging chemotherapeutic problem. The crucial role of angiogenesis in tumor growth has been widely recognized, and several reports have revealed that the combination treatment of the conventional chemotherapeutic drugs and anti-angiogenic agents exerted synergistic anti-cancerous effects. It has been reported that the clinically used angiotensin type- 1 receptor blocker (ARB) exerted potent anti-angiogenic activity. The aim of our current study was to examine the combination effect of gemcitabine (GEM), a widely used conventional chemotherapeutic drug against pancreas cancer, and losartan (Lo), an $\mathrm{ARB}$, on murine pancreatic tumor growth, especially in conjunction with angiogenesis. When used individually, GEM and Lo at clinically comparable low doses moderately suppressed pancreatic tumor development. The combination treatment with GEM and Lo exerted a marked inhibitory effect as compared with single agent treatments even after the tumor was fully established. Neovascularization and the expression of the vascular endothelial growth factor (VEGF), a central angiogenic factor, in the tumor were both markedly suppressed in a magnitude similar to the inhibitory effects against the tumor growth. Since both agents are widely used in the clinical practice, the combination regimen of GEM
\end{abstract}

Correspondence to: Dr Hitoshi Yoshiji, Third Department of Internal Medicine, Nara Medical University, Shijo-cho 840, Kashihara, Nara 634-8522, Japan

E-mail: yoshijih@naramed-u.ac.jp

Abbreviations: AT-II, angiotensin-II; ACE, angiotensin-converting enzyme; AT1-R, angiotensin type-1 receptor; ACE-I, ACE inhibitor; $\mathrm{ARB}$, angiotensin type-1 receptor blocker; EC, endothelial cell; HCC, hepatocellular carcinoma; GEM, gemcitabine; Lo, losartan; VEGF, vascular endothelial growth factor

Key words: VEGF, angiogenesis, gemcitabine, angiotensin, pancreatic tumor and Lo may represent a potential new therapeutic strategy for pancreatic cancer in the future.

\section{Introduction}

Exocrine pancreatic carcinoma is now the fifth leading cause of cancer in Japan, United States and Europe, with an overall 5 -year survival rate of less than $5 \%$ (1). The majority of patients are potential candidates for systemic treatment because the tumor is already unresectable at the time of diagnosis due to metastasis or the presence of locally advanced disease (2). Since 1997, gemcitabine (GEM) has been used for patients with advanced pancreatic cancer (3). Although GEM exerted a modest anti-tumor activity, it could achieve a significant improvement in the disease-related symptoms and prolong the survival of advanced pancreatic cancer patients. GEM produces a response rate of only $12-27 \%$ with a median survival of only 4-6 months, and it frequently induces drug resistance like most of the other conventional chemotherapeutic agents (3-5). New therapeutic approaches based on the biologic characteristics of this disease are required to improve the response rates and survival of patients with advanced pancreatic cancer.

It is now widely recognized that the growth of any solid tumor depends on angiogenesis. Without angiogenesis, the tumor ceases to grow even beyond a few millimeters in size; i.e., 'tumor dormancy' (6). Since inhibition of angiogenesis is now considered as a promising approach for cancer therapy, the efforts are currently directed worldwide at overcoming tumor angiogenesis (7). Although angiography in the clinical practice revealed that most pancreatic cancers are hypovascular or avascular, the human pancreatic cancer cells reportedly overexpress pro-angiogenic molecules such as the vascular endothelial growth factor (VEGF), a central key pro-angiogenic factor (8). In patients with pancreatic cancer, the intratumoral microvessel density proved to be an independent prognostic factor for survival in multivariate analysis $(9,10)$. Furthermore, in animal models, the anti-angiogenic compounds have proven very successful in inhibiting pancreatic tumor development (11-14). Collectively, angiogenesis also plays a pivotal role in the growth of pancreatic tumors. 
It has been reported that combination treatments of antiangiogenic agents and the conventional chemotherapeutic drugs at low doses exert a more potent inhibitory effect on tumor development than single-agent treatment (15). Since a long-term administration is required for anti-angiogenic treatment, selection of safe agents is necessary.

Angiotensin-II (AT-II) is an octapeptide produced mainly via proteolytic cleavage of its precursor angiotensin-I by angiotensin-converting enzyme (ACE), and is mainly coupled with angiotensin type-1 receptor (AT1-R). ACE inhibitor (ACE-I) and AT1-R blocker (ARB), that block the AT-II biological activities, are widely used as anti-hypertensive agents without serious side effects in the clinical practice. Several investigators including our group have shown that ACE-I and ARB possess strong anti-angiogenic activity, and that these agents could inhibit the growth of several types of tumors at clinically comparable low doses $(16,17)$. AT-II reportedly plays an important role in angiogenesis of pancreatic cancer through VEGF (18). Furthermore, it has been reported that ARB suppressed the growth of human pancreatic cancer cells in vitro (19).

In the current study, to evaluate the possible feasibility of future clinical application, we examined the combination effect of GEM and losartan (Lo), an ARB, at clinically comparable low doses on the murine pancreatic tumor development and angiogenesis, and we investigated the possible mechanisms involved.

\section{Materials and methods}

Compounds and cell lines. GEM and Lo were generously supplied by Eli Lilly Japan K.K. (Hyogo, Japan) and Banyu Pharmaceutical Co., Ltd. (Tokyo, Japan), respectively. The murine pancreatic cancer cell line, Pan02 (National Cancer Institute, Frederick, MD), was cultured in RPMI-1640 medium (Nacalai Tesque, Inc., Kyoto, Japan) supplemented with $10 \%$ (v/v) fetal bovine serum, $1 \%$ ampicillin/streptomycin, and $1 \%$ glutamine. The cells were grown in the media recommended by the respective suppliers.

Animal treatment. A total of 40 male 6-week-old C57BL/6 mice were purchased from Japan SLC, Inc. (Hamamatsu, Shizuoka, Japan). They were housed under controlled temperature conditions and relative humidity, with 10-15 air changes per hour (h) and light illumination for $12 \mathrm{~h}$ a day. To create the allograft model, $2.5 \times 10^{5}$ Pan02 cells were injected into the flank of C57BL/6 mice. After the tumor was fully established (the mean tumor volume was $200 \mathrm{~mm}^{3}$ on day 21), the mice were randomly divided into four groups $(n=10$ in each group). Group 1 (G1) consisted of untreated mice that served as a control group. Mice in G2 and G3 received GEM $(50 \mathrm{mg} / \mathrm{kg})$ twice a week via intraperitoneal injection and Lo $(30 \mathrm{mg} / \mathrm{kg} /$ day $)$ in the drinking water, respectively. The concentration of Lo in the drinking water was adjusted according to the water intake to maintain a constant daily dose of the drug. The doses of these agents in the current study were almost comparable to those used in the clinical practice $(20,21)$. To examine the combination effect of GEM and Lo, Lo was additionally administered to GEM group on day 49 (G4). The animals were allowed free access to food and water throughout the acclimation and experiment protocols. The tumor volume was measured twice a week, and the mice were sacrificed on day 73 after tumor cell implantation. All animal procedures were performed according to approved protocols and in accordance with the recommendations for the proper care and use of laboratory animals.

Immunohistochemistry. For determination of the in vivo angiogenesis, we employed immunohistochemical detection of platelet/EC adhesion molecule (PECAM/CD31), which is widely used as a marker of neovascularization, in frozen sections of tumors with the same size to avoid the necrotic effect of hypoxia as described previously (22). The immunostained microvessel length was assessed under x200-fold magnification. In each tumor sample, five areas showing the highest density of staining were selected for counting. In counting, the large vessels with a thick muscular wall or with a lumen $>50 \mu \mathrm{m}$ in diameter were excluded. These immunopositive vessels were evaluated with Adobe Photoshop and NIH image software as described previously (23).

Measurement of the VEGF expression in tumors. We measured the VEGF protein expression level in the tumor. Because different sizes of the tumor may cause different hypoxic conditions, which strongly induce VEGF (24), five tumors having the same size were chosen from each group. The tumor samples were prepared as described previously (16). After the protein concentration was equalized, the VEGF level was measured with an ELISA kit (R\&D Systems, Minneapolis, MN, USA) in accordance with the supplier's instructions.

Statistical analysis. To assess the statistical significance of inter-group differences in the quantitative data, Bonferroni's multiple comparison test was performed after one-way ANOVA. This was followed by Barlett's test to determine the homology of variance.

\section{Results}

Effect of GEM and Lo on tumor development. We first examined the effect of clinically comparable doses of GEM and Lo on the pancreatic tumor development. As shown in Fig. 1, single agent treatment with either GEM (G2) or Lo (G3) showed moderate inhibitory effect on the pancreatic tumor development as compared to the control group (G1) ( $<<0.05$, each). The inhibitory effect in G2 and G3 were almost of similar magnitude. Mice treated with GEM plus Lo (G4) showed a significant inhibition of the tumor volume as compared with those of G2 or G3 and G1 (p<0.05 and $<0.01$, respectively). The current doses of both agents did not affect the health status, such as body weights during the experiment in all experimental groups (data not shown).

Tumor neovascularization. To determine whether the inhibitory effect of GEM and Lo on the tumor development was accompanied by suppression of neovascularization, we next examined the tumor expression level of CD31. As shown in Fig. 2, the CD31-positive vessels in the tumors of either G2 or G3 were moderately fewer than those in the control 


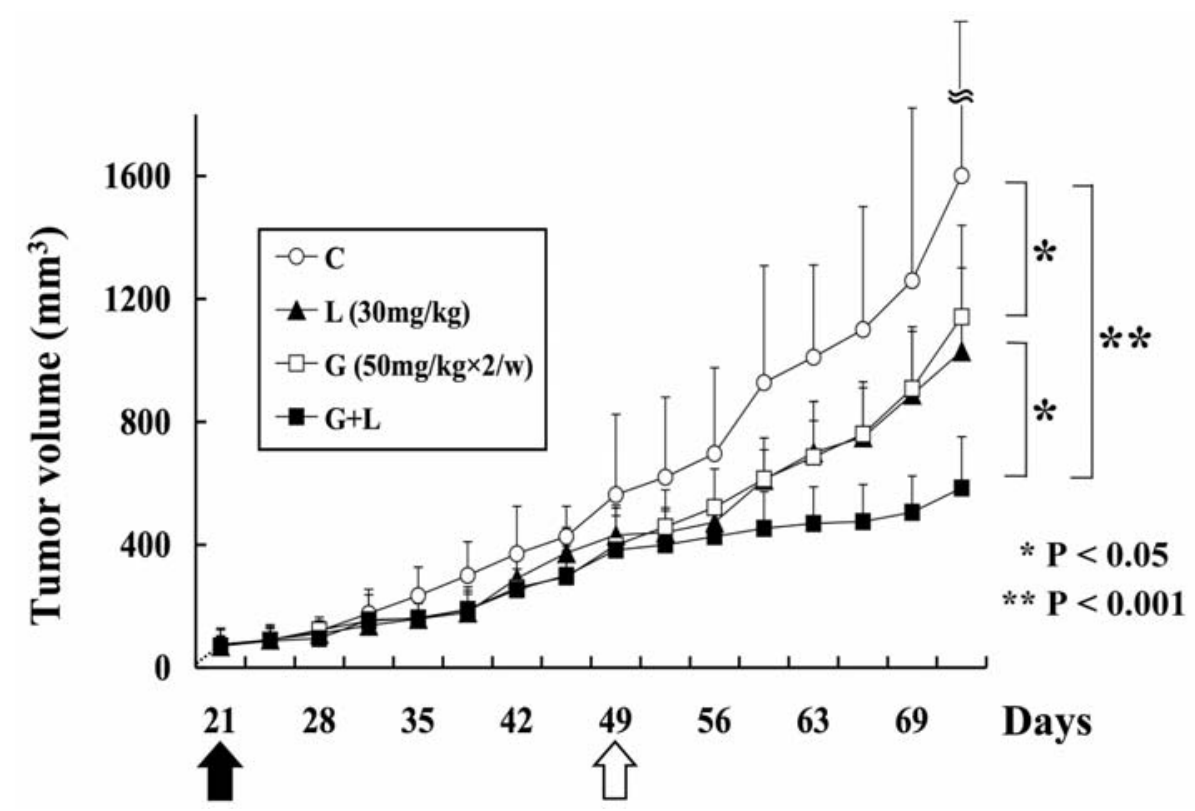

Figure 1. Effects of GEM and Lo on the pancreatic tumor development. Single treatment with either GEM ( $50 \mathrm{mg} / \mathrm{kg} / \mathrm{twice}$ a week, G2) or Lo (30 mg/kg/day, $\mathrm{G} 3)$ showed moderate inhibitory effect on the pancreatic tumor development as compared to the control group $(\mathrm{G} 1)(\mathrm{P}<0.05)$. The inhibitory effects in G2 and G3 were almost of similar magnitude. The mice treated with GEM plus Lo (G4) showed a significant inhibition in the tumor volume as compared with those of $\mathrm{G} 2$ or G3 and G1 ( $<<0.05$ and $<0.01$, respectively). The tumor volume was determined at the indicated time-points from day 21 (the mean tumor volume was $\left.200 \mathrm{~mm}^{3}\right)$. Each point represents the mean $\pm \mathrm{SD}(\mathrm{n}=10)$. Single and double asterisk indicate statistically significant differences between the indicated experimental groups ( $\mathrm{p}<0.05$ and $<0.01$, respectively). The white arrow indicates the time-point at which the combination treatment of GEM plus Lo started (day 49 ).
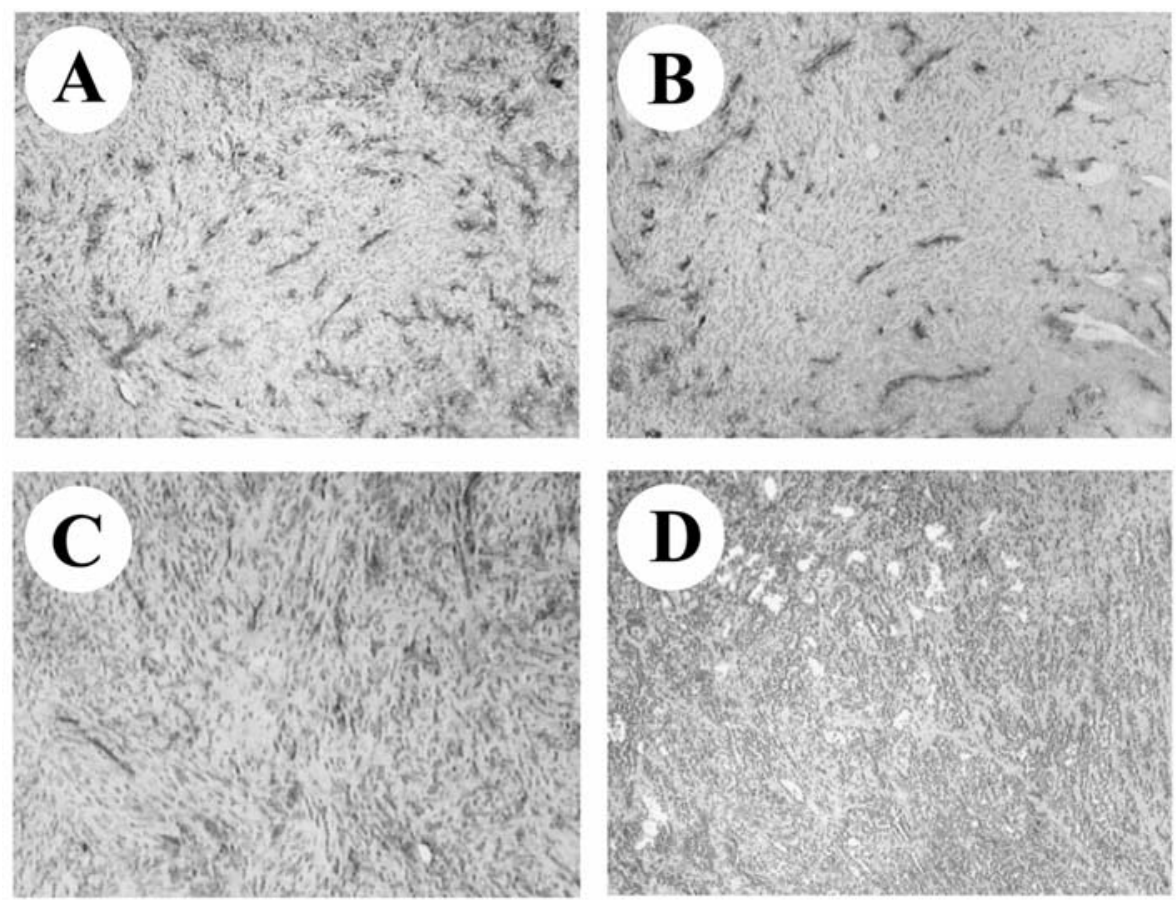

Figure 2. Immunohistochemical analysis of the CD31 expression in the tumor. Tumor vascularization was visualized by immunostaining of the CD31 vascular endothelial adhesion protein. (A), The control untreated group. (B), GEM-treated group. (C), Lo-treated group. (D), GEM and Lo combinationtreated group. The original magnifications are $\mathrm{x} 40$.

group; i.e., the results were similar to those of the pancreatic tumor development. The combination treatment of GEM plus Lo significantly attenuated the CD31-positive vessels in the tumor. We employed computer-assisted image analysis techniques to perform semi-quantification of neovascularization in the tumor as described previously (25). The semi- quantitative analysis revealed that the CD31-positive vessels in G2 or G3 were suppressed as compared to the control group ( $\mathrm{p}<0.05)$, and the combination treatment of GEM and Lo (G4) exerted a more potent inhibition of CD31-positive vessels in the tumor as compared to G2 and G3 $(\mathrm{p}<0.05)$ (Fig. 3). 


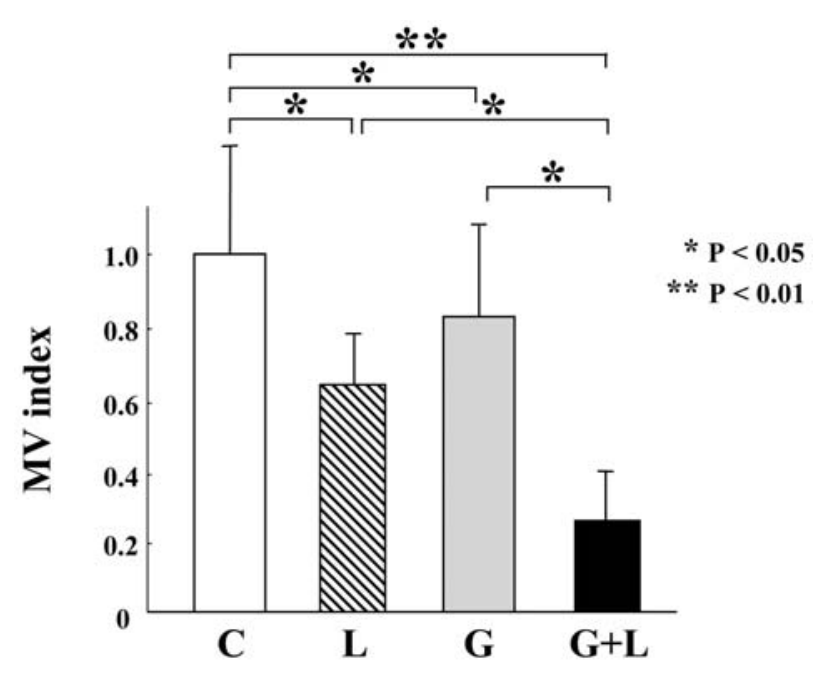

Figure 3. Semi-quantitative analysis of CD31-immunopositive vessels. The length of CD31-positive vessels in the tumor was measured by an image analysis system as described in Materials and methods section. The CD31positive vessels in the tumors of either G2 or G3 were moderately fewer than those in the control group, and these results matched those of the pancreatic tumor development. The combination treatment of GEM plus Lo significantly attenuated the CD31-positive vessels in the tumor. The data represent the mean $\pm \mathrm{SD}(\mathrm{n}=5)$. Single and double asterisk indicate statistically significant differences between the indicated experimental groups ( $\mathrm{p}<0.05$ and $<0.01$, respectively). C, the untreated control group. G and L, GEM- and Lo-treated group, respectively. G+L, GEM and Lo combinationtreated group.

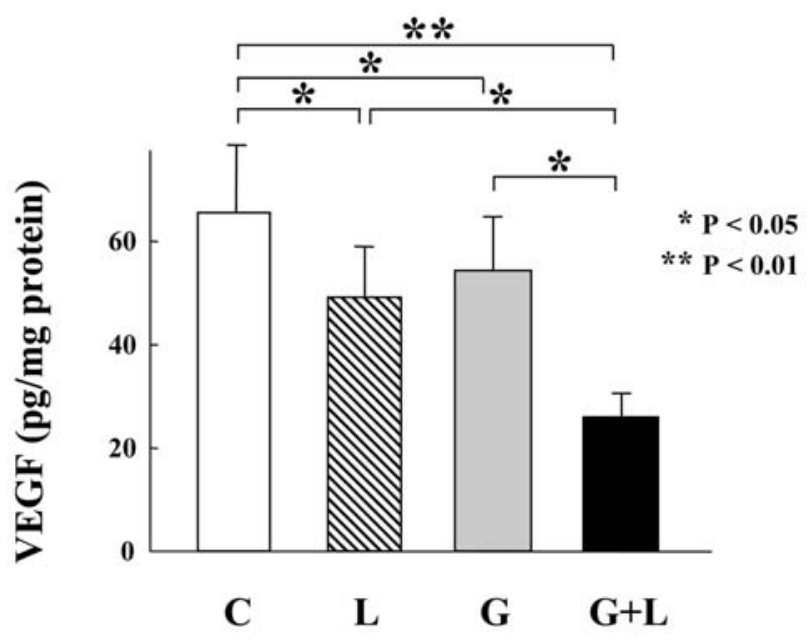

Figure 4. Effects of GEM and Lo on the VEGF expression in the tumor. The VEGF protein level was measured by ELISA as described in Materials and methods section. The VEGF expression in the tumor was suppressed by single agent treatment with GEM or Lo, and the combination treatment with GEM and Lo exerted a much stronger inhibition of VEGF as compared with either single agent. The data represent the mean $\pm \mathrm{SD}(\mathrm{n}=5)$. Asterisk indicates statistically significant difference as compared to the control group $(\mathrm{p}<0.05)$. $\mathrm{C}$, the untreated control group. G and L, GEM- and Lo-treated group, respectively. G+L, GEM and Lo combination-treated group.

Expression of VEGF in the tumor. To elucidate whether the suppressive effects of GEM and Lo on neovascularization were accompanied with inhibition of VEGF or not, we examined the VEGF expression of the tumor. As shown in Fig. 4, the VEGF expression in the tumor was suppressed by single agent treatment with GEM or Lo $(\mathrm{p}<0.05)$, and the combination treatment with GEM and Lo exerted a much stronger inhibition of VEGF as compared with either single agent $(\mathrm{p}<0.05)$. We also found the mRNA expression of VEGF in the tumor was suppressed by treatment with GEM and Lo (data not shown). The inhibitory effects of GEM and Lo on the VEGF expression were almost in parallel with those of inhibition of neovascularization in the tumor.

\section{Discussion}

In this study, we found that the single treatment with GEM or Lo exerted a modest inhibitory effect on the pancreatic tumor development along with suppression of VEGF-mediated angiogenesis, and the combination treatment of both agents exerted a more potent inhibitory effect as compared with either single agent. Therapies targeting the tumor vessels have proven very successful for cancer treatment in the experimental models (26). However, some recent studies have suggested that a treatment using single anti-angiogenic agent may not be sufficient to completely inhibit tumor angiogenesis. As such, the use of anti-angiogenic agents as monotherapy in treating the patients with advanced cancer has not yet shown any significant efficacy $(15,27)$. Significant compensatory up-regulation of several pro-angiogenic factors was observed after treatment with a single angiostatic agent. In contrast, treatment with combination angiostatic therapy significantly reduced the compensatory up-regulation $(15,28)$. Pre-clinical evidence indicated that combining antiangiogenic agents with a conventional chemotherapeutic agent therapy results in additive or even synergistic antitumoral effect $(12,29,30)$. Several studies have shown that the combination of newly developed anti-angiogenic agents (DC101, SU5416 and TNP-470) and GEM inhibited the tumor growth and angiogenesis synergistically in pancreatic cancer experimental models (31). Although several anti-angiogenic agents have already been employed in the clinical practice, these newly developed agents frequently induce severe side effects such as perforation of the gastrointestinal tract and hand-foot syndrome $(32,33)$. In the current study, we employed Lo as an anti-angiogenic agent. Lo is widely used currently without serious side effects in more than 100 countries, and its safety of administration to the patients with hypertension has already been proven. A noteworthy finding in this study was that the inhibitory effect of GEM and Lo could be observed at clinically comparable low doses of both agents. Furthermore, Lo was added after the tumor kept growing under the condition of GEM treatment. Since pancreatic tumors frequently exert drug resistance with single treatment of GEM in the clinical practice, we assume that the experimental condition in the current study was very similar to that of the patients with advanced GEM-resistant pancreatic cancer.

We observed that the inhibitory effects of GEM and Lo were associated with suppression of neovascularization and VEGF in the tumor. Of the numerous identified pro-angiogenic factors, VEGF appears to be a key factor in tumor angiogenesis, and VEGF is known to be a survival factor for the EC $(24,34)$. In the pancreatic tumor, VEGF was found to play an important role in supporting the aberrant growth of 
the tumor through maintaining its blood supply (35-37). Also, high levels of VEGF correlated with lymph node metastasis and poor prognosis in the patients with pancreatic cancer (38). The VEGF gene expression is regulated by several factors, including AT-II (18). Numerous tissues, including the pancreas (39-41), possess their own AT-II-generating systems that may finely tune specific function via paracrine/autocrine actions $(42,43)$. In pancreatic tumor cells, AT-II is a potent stimulator of the VEGF expression through AT1-R, and Lo suppressed the VEGF production in pancreatic tumor cell lines in a dose-dependent manner in vitro (18). Collectively, the suppressive effect of Lo on the VEGF expression in the tumor was likely mediated through both paracrine/autocrine mechanisms.

Similarly, GEM may also exert anti-tumoral effect through paracrine/autocrine mechanisms. Classically, GEM shows a marked anti-tumoral effect as a result of its cytotoxic action on the cancer cells (44). In addition to this cytotoxic activity, anti-angiogenic effect of GEM has recently been detected $(11,45)$. It has been reported that the EC were more sensitive to GEM than the pancreatic tumor cells in vitro, and that GEM inhibited neovascularization of the human pancreatic tumor in nude mice at very low-dose metronomic schedule (11). Taken together, it could be possible that coordination of these different biological activities of GEM and Lo via paracrine/autocrine mechanisms resulted in the in vivo combination marked tumoricidial effect. Further studies are required to elucidate the exact mechanism.

In summary, we have shown here that the combination treatment with GEM and Lo significantly inhibited the pancreatic tumor development along with suppression of VEGF-mediated neovascularization even after the tumor was fully established. Since both agents are currently widely used in the clinical practice, this combination regimen may represent a potential new therapeutic strategy for pancreatic cancer.

\section{References}

1. Jemal A, Murray T, Samuels A, Ghafoor A, Ward E and Thun MJ: Cancer statistics, 2003. CA Cancer J Clin 53: 5-26, 2003.

2. Li D, Xie K, Wolff R and Abbruzzese JL: Pancreatic cancer. Lancet 363: 1049-1057, 2004

3. Burris HA III, Moore MJ, Andersen J, Green MR, Rothenberg ML, Modiano MR, Cripps MC, Portenoy RK, Storniolo AM, Tarassoff P, Nelson R, Dorr FA, Stephens CD and von Hoff DD: Improvements in survival and clinical benefit with gemcitabine as first-line therapy for patients with advanced pancreas cancer: a randomized trial. J Clin Oncol 15: 2403-2413, 1997

4. Burris $\mathrm{H}$ and Storniolo AM: Assessing clinical benefit in the treatment of pancreas cancer: gemcitabine compared to 5fluorouracil. Eur J Cancer 33 (Suppl. 1): S18-S22, 1997.

5. Rothenberg ML, Moore MJ, Cripps MC, Andersen JS, Portenoy RK, Burris HA III, Green MR, Tarassoff PG, Brown TD, Casper ES, Storniolo AM and von Hoff DD: A phase II trial of gemcitabine in patients with 5-FU-refractory pancreas cancer. Ann Oncol 7: 347-353, 1996

6. Kerbel RS: Tumor angiogenesis: past, present and the near future. Carcinogenesis 21: 505-515, 2000.

7. Hanahan D and Weinberg RA: The hallmarks of cancer. Cell 100: $57-70,2000$

8. Kuwahara K, Sasaki T, Kuwada Y, Murakami M, Yamasaki S and Chayama K: Expressions of angiogenic factors in pancreatic ductal carcinoma: a correlative study with clinicopathologic parameters and patient survival. Pancreas 26: 344-349, 2003 .
9. Khan AW, Dhillon AP, Hutchins R, Abraham A, Shah SR, Snooks S and Davidson BR: Prognostic significance of intratumoural microvessel density (IMD) in resected pancreatic and ampullary cancers to standard histopathological variables and survival. Eur J Surg Oncol 28: 637-644, 2002.

10. Ikeda N, Adachi M, Taki T, Huang C, Hashida H, Takabayashi A, Sho M, Nakajima Y, Kanehiro H, Hisanaga M, Nakano H and Miyake M: Prognostic significance of angiogenesis in human pancreatic cancer. Br J Cancer 79: 1553-1563, 1999.

11. Laquente B, Lacasa C, Ginesta MM, Casanovas O, Figueras A, Galan M, Ribas IG, Germa JR, Capella G and Vinals F: Antiangiogenic effect of gemcitabine following metronomic administration in a pancreas cancer model. Mol Cancer Ther 7: 638-647, 2008.

12. Jia L, Zhang MH, Yuan SZ and Huang WG: Antiangiogenic therapy for human pancreatic carcinoma xenografts in nude mice. World J Gastroenterol 11: 447-450, 2005.

13. Zhang X, Galardi E, Duquette M, Lawler J and Parangi S: Antiangiogenic treatment with three thrombospondin-1 type 1 repeats versus gemcitabine in an orthotopic human pancreatic cancer model. Clin Cancer Res 11: 5622-5630, 2005.

14. Bruns CJ, Shrader M, Harbison MT, Portera C, Solorzano CC, Jauch KW, Hicklin DJ, Radinsky R and Ellis LM: Effect of the vascular endothelial growth factor receptor-2 antibody DC101 plus gemcitabine on growth, metastasis and angiogenesis of human pancreatic cancer growing orthotopically in nude mice. Int J Cancer 102: 101-108, 2002.

15. Kerbel RS: Clinical trials of antiangiogenic drugs: opportunities, problems, and assessment of initial results. J Clin Oncol 19: S45-S51, 2001.

16. Noguchi R, Yoshiji H, Kuriyama S, Yoshii J, Ikenaka Y, Yanase K, Namisaki T, Kitade M, Yamazaki M, Mitoro A, Tsujinoue H, Imazu H, Masaki T and Fukui H: Combination of interferon-beta and the angiotensin-converting enzyme inhibitor, perindopril, attenuates murine hepatocellular carcinoma development and angiogenesis. Clin Cancer Res 9: 6038-6045, 2003.

17. Yoshiji H, Kuriyama S and Fukui H: Angiotensin-I-converting enzyme inhibitors may be an alternative anti-angiogenic strategy in the treatment of liver fibrosis and hepatocellular carcinoma. Possible role of vascular endothelial growth factor. Tumour Biol 23: 348-356, 2002.

18. Arafat HA, Gong Q, Chipitsyna G, Rizvi A, Saa CT and Yeo CJ: Antihypertensives as novel antineoplastics: angiotensin-Iconverting enzyme inhibitors and angiotensin II type 1 receptor blockers in pancreatic ductal adenocarcinoma. J Am Coll Surg 204: 996-1006, 2007.

19. Fujimoto Y, Sasaki T, Tsuchida A and Chayama K: Angiotensin II type 1 receptor expression in human pancreatic cancer and growth inhibition by angiotensin II type 1 receptor antagonist. FEBS Lett 495: 197-200, 2001.

20. Schultz RM, Merriman RL, Toth JE, Zimmermann JE, Hertel LW, Andis SL, Dudley DE, Rutherford PG, Tanzer LR and Grindey GB: Evaluation of new anticancer agents against the MIA PaCa-2 and PANC-1 human pancreatic carcinoma xenografts. Oncol Res 5: 223-228, 1993.

21. Remuzzi A, Perico N, Amuchastegui CS, Malanchini B, Mazerska M, Battaglia C, Bertani T and Remuzzi G: Short- and long-term effect of angiotensin II receptor blockade in rats with experimental diabetes. J Am Soc Nephrol 4: 40-49, 1993.

22. Yoshiji H, Kuriyama S, Hicklin DJ, Huber J, Yoshii J, Miyamoto Y, Kawata M, Ikenaka Y, Nakatani T, Tsujinoue H and Fukui H: KDR/Flk-1 is a major regulator of vascular endothelial growth factor-induced tumor development and angiogenesis in murine hepatocellular carcinoma cells. Hepatology 30: 1179-1186, 1999

23. Yoshii J, Yoshiji H, Kuriyama S, Ikenaka Y, Noguchi R, Okuda $H$, Tsujinoue $H$, Nakatani T, Kishida H, Nakae D, Gomez DE, De Lorenzo MS, Tejera AM and Fukui H: The copper-chelating agent, trientine, suppresses tumor development and angiogenesis in the murine hepatocellular carcinoma cells. Int J Cancer 94: 768-773, 2001.

24. Shibuya M: Structure and function of VEGF/VEGF-receptor system involved in angiogenesis. Cell Struct Funct 26: 25-35, 2001.

25. Yoshiji H, Kuriyama S, Kawata M, Yoshii J, Ikenaka Y, Noguchi R, Nakatani T, Tsujinoue $\mathrm{H}$ and Fukui H: The angiotensin-i-converting enzyme inhibitor perindopril suppresses tumor growth and angiogenesis: possible role of the vascular endothelial growth factor. Clin Cancer Res 7: 1073-1078, 2001. 
26. Saif MW: Anti-angiogenesis therapy in pancreatic carcinoma. JOP 7: 163-173, 2006.

27. Carmeliet P and Jain RK: Angiogenesis in cancer and other diseases. Nature 407: 249-257, 2000.

28. Scappaticci FA, Smith R, Pathak A, Schloss D, Lum B, Cao Y, Johnson F, Engleman EG and Nolan GP: Combination angiostatin and endostatin gene transfer induces synergistic antiangiogenic activity in vitro and antitumor efficacy in leukemia and solid tumors in mice. Mol Ther 3: 186-196, 2001.

29. Muramaki M, Miyake H, Hara I, Kawabata G and Kamidono S: Synergistic inhibition of tumor growth and metastasis by combined treatment with TNP-470 and gemcitabine in a human bladder cancer KoTCC-1 model. J Urol 172: 1485-1489, 2004.

30. O'Reilly MS: The combination of antiangiogenic therapy with other modalities. Cancer J 8 (Suppl. 1): S89-S99, 2002.

31. Wang XF, Tu LF, Wang LH and Zhou JY: Inhibition of expression of vascular endothelial growth factor and its receptors in pulmonary adenocarcinoma cell by TNP-470 in combination with gemcitabine. J Zhejiang Univ Sci B 7: 837843, 2006.

32. Stathopoulos GP, Koutantos J, Lazaki H, Rigatos SK, Stathopoulos J and Deliconstantinos G: Capecitabine (Xeloda) as monotherapy in advanced breast and colorectal cancer: effectiveness and side-effects. Anticancer Res 27: 1653-1656, 2007.

33. Rouxel AM, Roguedas AM, Descourt R and Misery L: Handfoot syndrome: A new side effect of erlotinib. Ann Dermatol Venereol 135: 762-764, 2008.

34. Ferrara N: VEGF: an update on biological and therapeutic aspects. Curr Opin Biotechnol 11: 617-624, 2000.

35. Korc M: Pathways for aberrant angiogenesis in pancreatic cancer. Mol Cancer 2: 8, 2003.

36. Itakura J, Ishiwata T, Friess H, Fujii H, Matsumoto Y, Buchler MW and Korc M: Enhanced expression of vascular endothelial growth factor in human pancreatic cancer correlates with local disease progression. Clin Cancer Res 3: 1309-1316, 1997.
37. Itakura J, Ishiwata $\mathrm{T}$, Shen B, Kornmann $\mathrm{M}$ and Korc M: Concomitant over-expression of vascular endothelial growth factor and its receptors in pancreatic cancer. Int J Cancer 85: 27-34, 2000.

38. Buchler P, Reber HA, Buchler MW, Friess H and Hines OJ: VEGF-RII influences the prognosis of pancreatic cancer. Ann Surg 236: 738-749, 2002.

39. Tahmasebi M, Puddefoot JR, Inwang ER and Vinson GP: The tissue renin-angiotensin system in human pancreas. J Endocrinol 161: 317-322, 1999.

40. Leung PS and Chappell MC: A local pancreatic reninangiotensin system: endocrine and exocrine roles. Int J Biochem Cell Biol 35: 838-846, 2003.

41. Ohta T, Amaya K, Yi S, Kitagawa H, Kayahara M, Ninomiya I, Fushida S, Fujimura T, Nishimura G, Shimizu K and Miwa K: Angiotensin converting enzyme-independent, local angiotensin II-generation in human pancreatic ductal cancer tissues. Int J Oncol 23: 593-598, 2003.

42. Campbell DJ and Habener JF: Angiotensinogen gene is expressed and differentially regulated in multiple tissues of the rat. J Clin Invest 78: 31-39, 1986

43. Campbell DJ: Circulating and tissue angiotensin systems. J Clin Invest 79: 1-6, 1987.

44. Storniolo AM, Allerheiligen SR and Pearce HL: Preclinical, pharmacologic, and phase I studies of gemcitabine. Semin Oncol 24: S7-2-S7-7, 1997.

45. Amoh Y, Li L, Tsuji K, Moossa AR, Katsuoka K, Hoffman RM and Bouvet M: Dual-color imaging of nascent blood vessels vascularizing pancreatic cancer in an orthotopic model demonstrates antiangiogenesis efficacy of gemcitabine. J Surg Res 132: 164-169, 2006. 